\title{
A STUDY OF MULTIORGAN DYSFUNCTION IN PATIENTS WITH DENGUE AND ITS CLINICO- HEMATOLOGICAL CORRELATION WITH SEVERITY
}

\author{
JADUMANI NAYAK ${ }^{1}$, SUDESHNA BEHERA ${ }^{2 *}$, SHRIKANTA K SWAIN ${ }^{1}$, SMARAKA R PANDA ${ }^{3}$ \\ ${ }^{1}$ Department of Medicine, S.C.B. Medical College, Cuttack, Odisha, India. ${ }^{2}$ Department of Biochemistry, IMS and SUM Hospital, Siksha \\ “0” Anusandhan University, Bhubaneswar, Odisha, India. ${ }^{3}$ Department of Nephrology, S.C.B Medical College, Cuttack, Odisha, India. \\ Email: 4sudeshna@gmail.com
}

Received: 23 September 2016, Revised and Accepted: 03 November 2016

ABSTRACT

Objective: The aim was to study of multiorgan dysfunction in patients with dengue and to correlate the severity and clinical outcome with different hematological parameters.

Methods: All suspected cases of dengue admitted to medicine department within 1 year were evaluated and classified as dengue fever (DF), dengue hemorrhagic fever (DHF), and dengue shock syndrome (DSS).

Results: Out of 150 cases, 108 cases were classified as DF, 31 cases as DHF and 11 cases as DSS. Liver involvement was seen in $97.33 \%$ of cases. Jaundice was observed in 10 cases (6.6\%), abnormal aspartate transaminase in 146 (97.33\%) cases, and abnormal alanine transaminase in 126 (84\%) cases. Bleeding manifestations was reported in 61 patients (40.6\%). The presence of gastrointestinal bleeding in 17 patients (11.3\%) was related to severe disease. Thrombocytopenia was present in 25\% of DF cases, and in all cases of DHF and DS. Among these, prothrombin time (PT) was prolonged in 10 cases and activated partial thromboplastin time (aPTT) in 47 patients. Serum fibrinogen was low in $28.57 \%$ of patients. Multiorgan dysfunction was observed in the form of simultaneous hepatic and renal dysfunction in $3.33 \%$ of cases, hepatic and central nervous system dysfunction in $2.66 \%$ of cases, hepatic and cardiac dysfunction in $2 \%$ of cases, and all the organ system dysfunction in $0.67 \%$ of cases.

Conclusion: The extent of increase of liver enzymes, PT, aPTT and fall of serum fibrinogen, and degree of thrombocytopenia correlated with disease severity. Acute renal failure, encephalitis, and myocarditis are manifestations of severe form of dengue. Major organ involvement may occur in simple DF also.

Keywords: Dengue fever, Dengue hemorrhagic fever, Dengue shock syndrome.

(c) 2017 The Authors. Published by Innovare Academic Sciences Pvt Ltd. This is an open access article under the CC BY license (http://creativecommons. org/licenses/by/4. 0/) DOI: http://dx.doi.org/10.22159/ajpcr.2017.v10i2.15332

\section{INTRODUCTION}

Dengue is one of the major re-emerging viral infections. In recent years, dengue has become a major global public health concern [1]. Approximately, 2.5 billion people ( $40 \%$ of the world's population), living mainly in urban areas of tropical and subtropical regions, are estimated to be at risk of acquiring dengue infection. The WHO estimates that 50-100 million infections occur yearly including 500,000 dengue hemorrhagic fever (DHF) cases and 22,000 deaths, mostly among children. While dengue is endemic in more than 100 countries, most cases are reported from South-East Asia and the western Pacific regions [2]. The South-East Asia Region has become hyperendemic with regular reporting of dengue cases since 2000 . The maximum number of cases $(355,525)$ and deaths (1982) were recorded during 2010 . Since then, a declining trend is being reported [3]. First recorded case in India was in the year of 1812. Although several measures were taken to prevent and control it, recurrent outbreaks have been reported in India and it was observed that clinical manifestations during recent outbreaks are slightly different from the previous ones [4]. Odisha state in 2010, enrolled its name for the first time in the list of states showing mortality due to dengue infection, with the reporting of 25 cases and five deaths [5]. Maximum number of cases during 2014 have been reported from Odisha (5079). Maximum number of death due to dengue infection has been reported from Kerala, Madhyapradesh, and Odisha (9 in each state). Hence, the elucidation of the exact clinical profile is very much required for proper patient management and saving of life.
Considering the rampant outbreaks of dengue in India, particularly in this eastern zone and studying various literatures regarding the varied clinical manifestations of dengue patients in recent years, this study was designed to evaluate the clinical outcomes of dengue patients and their various hematological parameters and finally to correlate these with the severity.

\section{METHODS}

The study included 150 patients with dengue virus infection admitted to the department of Medicine, Sriram Chandra Bhanja Medical College, Cuttack within 1 year. Inclusion criteria was all suspected cases of dengue as defined under National Vector Borne Disease Control Programme (NVBDCP), Government of India, who were tested positive for enzyme-linked immunosorbent assay (ELISA) based nonstructural protein 1 (NS1) antigen and immunoglobulin M (IgM) capture ELISA (MAC ELISA). Various exclusion criteria include patients with malaria or known positive serology for viral hepatitis, patients with chronic liver diseases, diabetes mellitus with nephropathy or cardiomyopathy, chronic kidney disease, and collagen vascular diseases.

The Institute Ethics Committee was approached for the Ethical Clearance. An informed consent was obtained from all the participants before collecting the blood samples.

Patients were evaluated and classified using the case definitions recommended by the WHO (1997) under NVBDCP, Government of 
India as dengue fever (DF), DHF, and dengue shock syndrome (DSS). Various laboratory investigations and systemic investigations were carried out in all patients. All statistical analysis was performed in SPSS software version 20. Fischer's exact test and student's unpaired $t$-test were performed for significance of difference of proportions and means between two groups, respectively. Mann-Whitney test was used for significance of difference in means for non-Gaussian data.

\section{Observations}

In our study, out of 150 cases, total number of males were 106 (71\%), whereas females were $44(29 \%)$ in our study. Male to female ratio was 2.4:1. Out of 150 patients, 83 (55.33\%) were positive for NS1 ELISA, $50(33.33 \%)$ positive for IgM ELISA, and 17 (11.33\%) were positive for both. Fever was the main presenting symptom (100\%) in all our patients followed by headache (94\%), myalgia (90.6\%), nausea/ vomiting (80\%), retro-orbital pain (42\%), bleeding manifestations (40.6\%), rash (35.3\%), arthralgia (26.6\%), abdominal pain $(23.3 \%)$, and diarrhea $(14.6 \%)$. The clinical examination revealed jaundice in 10 patients $(6.6 \%)$. Ultrasonographic evaluation revealed hepatomegaly in 62 patients $(41.3 \%)$. Prothrombin time (PT) and activated partial thromboplastin time (aPTT) were high in patients with bleeding manifestations and even higher in patients with gastrointestinal (GI) hemorrhage. Non survivors showed PT, aPTT of more than 1 minute. aPTT was significantly abnormal in more percentage of patients with bleeding manifestations and GI hemorrhage than PT. Serum fibrinogen was low in $28.57 \%$ of patients with bleeding manifestations, $50 \%$ of those with GI hemorrhage and all patients of nonsurvivors.

Out of 150 cases, acute renal failure (ARF) was present in 4\% (n=6) of cases, Proteinuria in $22 \%(n=33)$ cases and hematuria in $4 \%(n=6)$ cases. Renal Complications are more manifested in severe dengue (DHF and DSS). Out of 6 patients of dengue with ARF, 2 patients died with multiorgan dysfunction. One patient had ARF, proteinuria and hematuria. Another had both ARF and proteinuria. Both the patients had Jaundice, prolonged PT, aPTT with low serum fibrinogen. Out of the two, one had dengue encephalitis.

Out of all the dengue patients, 3 patients with severe dengue had following features arrhythmia, myocarditis, hypotension, and pulmonary edema. Out of the 3 patients, one had DHF and two had DSS. All the 3 patients had abnormal electrocardiogram reading. Two patients had atrial fibrillation and one patient had features of $1^{\text {st }}$ degree heart block. Myocarditis was present in $33.3 \%$ of patients, hypotension in $66.67 \%$ of patients and pulmonary edema in $66.67 \%$ of patients in severe dengue, presenting with cardiovascular manifestations. Twodimensional echocardiographic evaluation was done in all 3 patients. 2 patients had normal Echo study and one had features of myocarditis. A fatal outcome was reported in a case with DSS with myocarditis.

Out of 150 dengue patients, only 5 patients (3.33\%) presented with dengue encephalitis. Most of the patients $(n=4)$ belonged to severe form of dengue (DHH and DSS). Apart from fever and headache, altered sensorium $(60 \%)$ and convulsion $(40 \%)$, remain the most common presentation of dengue encephalitis $(n=5)$. Cerebral edema has been primary finding in computed tomography scan and magnetic resonance imaging (MRI). MRI revealed cerebral edema in (3/5) majority of patients.

\section{DISCUSSION}

This prospective study was conducted to assess multiorgan dysfunction in dengue and the association of clinical outcomes and different hematological parameters with the severity of 150 serologically confirmed cases of dengue infection during 1 year in the Department of Medicine, SCB Medical College, and Cuttack.

Males were predominantly infected in each age group. The male predominance has also been reported in several other studies [6]. The mean age of patients in our study was 32.4 years which is similar to other studies where higher age groups were observed of developing symptomatic disease than younger groups [7-9] and contradictory to the study by Raut and Patil where they revealed children $<10$ years were affected more [10].

In our study, $72 \%$ of patients were classified as DF, $21 \%$ as DHF and $7 \%$ as DSS. A very similar study from eastern India by Chatterjee et al. on 180 serologically confirmed dengue cases showed an incidence of $71 \%$ and 29\% of DF and DHF/DSS cases respectively [11]. Chhina et al. [12] and Makroo et al. [13] reported 19\% of cases of DHF/DSS cases.

Fever was the main presenting symptom in all our patients followed by headache (94\%), myalgia (90.6\%). Sedhain et al. in their study of 414 patients with dengue infection in Nepal reported a profile of clinical manifestations akin to our cases [14]. Abdominal pain in our study was present in $35 / 150(23.3 \%)$ cases. This is in agreement with the study of Chhina et al. who similarly reported pain abdomen in $20 \%$ of cases [12]. Our study showed hepatomegaly in $41.3 \%$ of cases. Recent studies suggest that hepatomegaly is present at between 50 and $100 \%$ of cases [15] while some have documented lower rate of hepatomegaly like $24 \%$ by Itha et al. [16].

Bleeding in dengue is due to thrombopathy, coagulopathy, and vasculopathy. Bleeding manifestations in our study was reported in $40.6 \%$ of cases. Bleeding into different sites has been observed in most studies [17-19].

Thrombocytopenia (total platelet count [TPC] $<100,000 / \mathrm{cm}^{2}$ ) was present in $25 \%$ of DF cases, whereas in all cases of DHF and DSS. None had GI hemorrhage. Sedhain et al. also reported a platelet count of $<50,000 / \mathrm{cm}^{2}$ in $9.73 \%$ of DF patients, which was similar to our finding [14]. Thrombocytopenia is a major finding in a significant number of cases of DHF/DSS and increases with severity of disease.

In our study, Table 1 showed abnormal aspartate transaminase (AST) was present in $96.29 \%$ of DF cases and in all patients with DHF and DSS in our study. Similarly, abnormal alanine transaminase (ALT) was present in $79.62 \%$ of DF cases, $93.54 \%$ of DHF cases and in all patients of DSS. Total bilirubin, AST, ALT, alkaline phosphatase (ALP) were significantly higher in DHF and DSS groups than DF category. The AST levels in dengue infection tend to be greater than ALT levels. This differs from the pattern in viral hepatitis but is similar to that seen in alcoholic hepatitis. The exact cause of this difference is unknown, but it has been suggested that it may be due to excess release of AST from damaged monocytes during dengue infection [20]. Ageep in 2012 reported high AST and ALT levels in $86 \%$ and $82 \%$ of patients, respectively [21]. However, Wong and Shen reported that AST abnormality was predominantly higher than ALT, $91 \%$ and $72 \%$, respectively [22].

Serum albumin was significantly lower in severe dengue infections (DHF/DSS) than patients with DF. Hypoalbuminaemia is a result of impairment of the synthetic function of liver as it is the target organ of dengue virus. Hypoalbuminemia is more severe in severe dengue [23].

In Saudi Arabia, Khan et al. found an association between high AST level and complications in dengue infection [24]. A report from India by Shah pointed to a high mortality in dengue patients with hepatitis and encephalopathy [25]. Ageep noticed relation between the degree of liver damage and the presence of complications [21]. In $71 \%$ of the patients having DHF, severe degree of liver damage was observed. He suggested that the deranged liver functions may participate in the causation of bleeding in these patients. Severe degree of liver injury also was found in $80 \%$ of DSS. All of the patients having encephalopathy had severe liver damage. He proposed encephalopathy may be due to fulminant hepatic failure or a high level of the virus that directly damage the brain. Involvement of the kidneys was also related to the severity of liver damage.

According to Table 2, PT, aPTT were high in our patients with bleeding manifestations and even higher in patients with GI hemorrhage. Non survivors showed PT, aPTT of more than 1 minute. This observation is similar to earlier studies by Hathirat et al. that aPTT is more frequently 
Table 1: Comparison of laboratory parameters among patients with DF, DHF and DSS

\begin{tabular}{|c|c|c|c|c|c|}
\hline Laboratory category variables & DF $(n=108)$ & DHF (n=31) & $\mathbf{p}^{¥}$ value & DSS (n=11) & $\mathbf{p}^{¥ ¥}$ value \\
\hline $\mathrm{PCV}^{*}$ & $38.32 \pm 0.46$ & $33.13 \pm 0.85$ & $<0.001$ & $35 \pm 3.02$ & 0.055 \\
\hline TPC* & $1.42 \pm 0.06$ & $0.59 \pm 0.03$ & $<0.001$ & $0.53 \pm 0.08$ & $<0.001$ \\
\hline Total bilirubin* & $1 \pm 0.04$ & $1.87 \pm 0.34$ & $<0.001$ & $2.89 \pm 0.59$ & $<0.001$ \\
\hline AST* $^{*}$ & $151.5 \pm 17.56$ & $316.7 \pm 9.68$ & $<0.001$ & $509.7 \pm 0.69$ & $<0.001$ \\
\hline ALT* & $110.3 \pm 16.09$ & $182.2 \pm 4.67$ & $<0.001$ & $397.5 \pm 67.3$ & $<0.001$ \\
\hline ALP* & $161.8 \pm 7.07$ & $205.1 \pm 0.11$ & 0.024 & $211 \pm 34.86$ & 0.526 \\
\hline Sr. Albumin* & $3.42 \pm 0.03$ & $3.15 \pm 0.03$ & $<0.001$ & $2.53 \pm 0.11$ & $<0.001$ \\
\hline
\end{tabular}

*Data are expressed as the mean \pm SEM. ${ }^{¥}$ p value between DF and DHF groups. ${ }^{¥ \neq}$ p value between DF and DSS groups. DF: Dengue fever, DHF: Dengue hemorrhagic fever, DSS: Dengue shock syndrome, PCV: Packed cell volume, TPC: Total platelet count

Table 2: Comparison of coagulation and hematological parameters in patients with DF, DHF, and DSS

\begin{tabular}{|c|c|c|c|c|c|}
\hline Parameters & DF $(n=108)(\%)$ & DHF (n=31) (\%) & $\mathbf{p}^{\#}$ value & DSS n=11 (\%) & $\mathbf{p}^{\# \#}$ value \\
\hline PT $(n=61)^{*}$ Number $(\%)$ of patients with $>$ ULN & $0 / 19(0)$ & $4 / 31(12.9)$ & 0.283 & $6 / 11(54.5)$ & $<0.001$ \\
\hline aPTT $(n=61)^{*}$ Number $(\%)$ of patients with $>$ ULN & $6 / 19(31.6)$ & $30 / 31(96.8)$ & $<0.001$ & $11 / 11(100)$ & $<0.001$ \\
\hline Sr. Fibrinogen $(n=42) *$ Number $(\%)$ of patients with $<$ LLN & $0 / 7(0)$ & $5 / 25(20)$ & 0.560 & $7 / 10(70)$ & 0.009 \\
\hline $\mathrm{TPC}<50,000$ & $6 / 108(5.5)$ & $12 / 31(38.7)$ & $<0.001$ & $6 / 11(54.5)$ & $<0.001$ \\
\hline $\mathrm{PCV} \geq 20$ & $0 / 108(0)$ & $5 / 31(16.1)$ & $<0.001$ & $6 / 11(54.5)$ & $<0.001$ \\
\hline
\end{tabular}

*Number of patients in which the test was available. \#p value between DF and DHF groups. \#\#p value between DF and DSS groups. ULN: Upper limit of normal,

LLN: Lower limit of normal. (Normal upper limit of PT-16.7 seconds and for aPTT-34.6 seconds. Normal serum fibrinogen-1.1-4.2 g/L). DF: Dengue fever, DHF: Dengue

hemorrhagic fever, DSS: Dengue shock syndrome, PCV: Packed cell volume, TPC: Total platelet count

Table 3: Comparison of clinical parameters among patients with dengue infection

\begin{tabular}{|c|c|c|c|c|c|}
\hline Clinical category variables & DF $n=108(\%)$ & DHF $n=31(\%)$ & $\mathbf{p}^{\mp}$ value & DSS $n=11(\%)$ & $\mathbf{p}^{¥ ¥}$ value \\
\hline Rash (\%) & $47 / 108(43.5)$ & $4 / 31(12.9)$ & 0.001 & $2 / 11(18.2)$ & 0.121 \\
\hline Muco-cutaneous bleeding (\%) & $19 / 108(17.6)$ & $29 / 31(93.5)$ & $<0.001$ & $10 / 11(90.9)$ & $<0.001$ \\
\hline GI hemorrhage (\%) & $0 / 108(0)$ & $12 / 31(38.7)$ & $<0.001$ & $5 / 11(45.4)$ & $<0.001$ \\
\hline Jaundice $(\%)$ & $2 / 108(1.9)$ & $4 / 31(12.9)$ & 0.022 & $4 / 11(36.4)$ & $<0.001$ \\
\hline $\operatorname{ARF}(\%)$ & $1 / 108(0.925)$ & $2 / 31(6.451)$ & $<0.001$ & $3 / 11(27.27)$ & $<0.001$ \\
\hline Hematuria & $0(0)$ & $4 / 31(12.95)$ & $<0.002$ & $2 / 11$ (18.19) & $<0.001$ \\
\hline Proteinuria & $4 / 108(3.7)$ & $20 / 31(64.5)$ & $<0.001$ & $9 / 11(81.81)$ & $<0.001$ \\
\hline Myocarditis & $0(0)$ & $0(0)$ & $<0.001$ & $1 / 11(9.09)$ & $<0.001$ \\
\hline Encephalopathy & $1(0.925)$ & $2(6.5)$ & $<0.001$ & $2(18.18)$ & $<0.001$ \\
\hline
\end{tabular}

*Data are expressed as the mean \pm SEM. ${ }^{*}$ p value between DF and DHF groups. ${ }^{¥ \neq}$ p value between DF and DSS groups. DF: Dengue fever, DHF: Dengue hemorrhagic fever,

GI: Gastrointestinal, ARF: Acute renal failure

Table 4: Comparison of renal complications and cardiac complications in DF, DHF, and DSS cases

\begin{tabular}{|c|c|c|c|c|c|c|c|}
\hline \multicolumn{4}{|c|}{ Comparison of renal complications } & \multicolumn{4}{|c|}{ Comparison of cardiac complications } \\
\hline Clinical category & DF $n=108(\%)$ & DHF (n=31) (\%) & DSS $(n=11)(\%)$ & Clinical category $(n=3)$ & $\mathrm{DF}(\mathrm{n}=\mathbf{0})$ & DHF $(n=1)$ & DSS $(n=2)$ \\
\hline $\operatorname{ARF}(n=6)$ & $1(0.925)$ & $2(6.45)$ & $3(27.23)$ & Arrhythmia & 0 & 1 & 2 \\
\hline Proteinuria (n=33) & $4(3.70)$ & $20(64.5)$ & $9(81.81)$ & Myocarditis & 0 & 0 & 1 \\
\hline \multirow[t]{2}{*}{ Hematuria $(n=6)$} & $0(0)$ & $4(12.9)$ & $2(18.19)$ & Hypotension & 0 & 0 & 2 \\
\hline & & & & Pulmonary edema & 0 & 1 & 1 \\
\hline
\end{tabular}

DF: Dengue fever, DHF: Dengue hemorrhagic fever, ARF: Acute renal failure

abnormal than PT suggesting that a defect occurs in the intrinsic pathway of coagulation [26]. An analysis of the linear correlation and regression between the levels of AST/ALT and aPTT show a strong association between AST/ALT elevation and aPTT prolongation in DHF and DSS patients. This suggests dysfunction of the damaged liver might be responsible for the decreased synthesis of specific factors in the intrinsic pathway. Increased factor consumption as indicated by high level of tissue plasminogen activator in previous studies is also associated with aPTT prolongation, but in a less significant manner. Low serum fibrinogen level of our study is in agreement with various other studies [27].

Table 3 reported GI hemorrhage was a consistent finding in our patients with severe dengue. This correlated with higher bilirubin, AST, ALT, ALP, prolonged PT, aPTT, low TPC, low albumin, and low fibrinogen.
In our study of 150 patients (Table 4), 5 cases of ARF had severe dengue (DHF and DSS), but one case of ARF was detected in the absence of hemorrhage or shock. Out of $33(22 \%)$ patients with proteinuria, $29(87.87 \%)$ patients had severe dengue. All of the patients $(n=6)$ (4\%) with hematuria had severe dengue. $12.9 \%$ of DHF patients had hematuria. The study by Horvath et al. also showed proteinuria in $74 \%$ of patients with severe dengue and hematuria in $12.5 \%$ of patients with DHF [28]. The study by Nair et al. in 2012 presented a patient with DF with ARF requiring hemodialysis in the absence of features of hemorrhage or shock, as seen in one case in our study [29].

In our study, 3 patients with severe dengue had cardiological manifestations. Out of which, one had DHF and two had DSS. A fatal outcome was reported in a case as DSS with myocarditis and encephalitis. The patient died from multiorgan dysfunction. 
Five patients developed neurological manifestation in the form of altered sensorium $(n=3)$ and convulsion $(n=2)$. Overall, 4 cases of encephalitis had severe dengue $(80 \%)$, but one case was detected in DF, in the absence of hemorrhage or shock. The study by PuccioniSohler et al., showed neurological involvement in $4.5 \%$ of confirmed dengue cases [30]. MRI revealed cerebral edema in patients with dengue encephalitis. The study by Cam et al., showed, out of 18 patients with dengue encephalitis, 12 (66\%) had cerebral edema, no change in 4 patients, and only the minority $(n=2)$ showed specifically scattered focal lesions in MRI [31]. The study by Solomon et al. showed dengue presenting as acute encephalitic syndrome without manifestation of the disease, which we found in one case in our study [32]. The two patients with encephalopathy, one each belonged to DHF and DSS group, died due to multiorgan dysfunction.

Limitations of our study mainly include leaving out all outpatient departmental cases. Relevant data were collected only from the admitted patients. Furthermore, there was lacking of entomological data.

\section{CONCLUSION}

This may be concluded from our study that, the extent of rise of liver enzymes, PT, aPTT, the extent of fall of serum fibrinogen, and the degree of thrombocytopenia, correlated with disease severity. ARF, encephalitis and myocarditis are manifestations of severe form of dengue with poor clinical outcome. Major organ involvement may occur in simple DF also. This study will be helpful for the clinicians to have a better understanding regarding clinical manifestations, outcomes, and complications of dengue infection. Although some known clinical features are still manifesting, but various atypical features have also reported in different epidemics. Hence always a continuous and timely sero-epidemiological surveillance is required in endemic areas of dengue with proper interventions to minimize its complications, outbreak, and mortality.

\section{REFERENCES}

1. Padhi S, Dash M, Panda P, Parida B, Mohanty I, Sahu S, et al. A three year retrospective study on the increasing trend in seroprevalence of dengue infection from southern Odisha, India. Indian J Med Res 2014;140:660-4

2. World Health Organization, Global Alert and Response (GAR). Impact of Dengue. Available from: http://www.who.int/csr/disease/dengue/ impact/en/. [Last accessed on 2013 Feb 19].

3. WHO. Dengue Bulletin. World Health Organization, 2012. Available from: http://www.wpro.who.int/mvp/epidemiology/dengue/Dengue Bulletin Vol36.pdf. [Last assessed on $2016 \mathrm{Jul}$ 01].

4. Seema A, Singh V, Kumar S, Kumar A, Dutta S. The changing clinical spectrum of dengue fever in the 2009 epidemic in North India: A tertiary teaching hospital based study. J Clin Diagn Res 2012;6(6):999-1002.

5. Government of India, Health and Family Welfare Department, National Vector Borne Disease Control Programme (NVBDCP): Dengue cases and deaths in the country since 2007.e2012. Available from: http:// www.nvbdcp.gov.in/dencd.html. [Last assessed on 2012 Dec 05].

6. Anker M, Arima Y. Male-female differences in the number of reported incident dengue fever cases in six Asian countries. Western Pac Surveill Response J 2011;2(2):17-23.

7. Thai KT, Nishiura H, Hoang PL, Tran NT, Phan GT, Le HQ, et al. Age specificity of clinical dengue during primary and secondary infections. PLoS Negl Trop Dis 2011;5(6):e1180.

8. Egger JR, Coleman PG. Age and clinical dengue illness. Emerg Infect Dis 2007; $13: 924-5$.

9. Isalkar U. Most dengue cases in 14-45 age group: PMC analysis. Pune,
India: The Times of India; 2013.

10. Raut S, Patil S. Dengue in and around Nagpur-central India. J Evol Med Dent Sci 2012;1(5):853.

11. Chatterjee N, Mukhopadhyay M, Ghosh S, Mondol M, Das C, Patar K. An observational study of dengue fever in a tertiary care hospital of eastern India. J Assoc Physicians India 2014;62:224-7.

12. Chhina RS, Goyal O, Chhina DK, Goyal P, Kumar R, Puri S. Liver function tests in patients with dengue viral infection. Dengue Bull 2008;32:110-7.

13. Makroo RN, Raina V, Kumar P, Kanth RK. Role of platelet transfusion in the management of dengue patients in a tertiary care hospital. Asian J Transfus Sci 2007;1:4-7.

14. Sedhain A, Bhattarai GR, Adhikari S, Shrestha B, Sapkota A. Liver involvement associated with dengue infection during a major outbreak in central Nepal. J Adv Intern Med 2013;02(02):42-6.

15. Wahid SF, Sanusi S, Zawawi MM, Ali RA. A comparison of the pattern of liver involvement in dengue hemorrhagic fever with classic dengue fever. Southeast Asian J Trop Med Public Health 2000;31:259-63.

16. Itha S, Kashyap R, Krishnani N, Saraswat VA, Choudhuri G, Aggarwal R. Profile of liver involvement in dengue virus infection. Natl Med J India 2005;18:127-30.

17. Kumar A, Rao CR, Pandit V, Shetty S, Bammigatti C, Samarasinghe CM. Clinical manifestations and trend of dengue cases admitted in a tertiary care hospital, Udupi district, Karnataka. Indian J Community Med 2010;35:386-90.

18. Karoli R, Fatima J, Siddiqi Z, Kazmi KI, Sultania AR. Clinical profile of dengue infection at a teaching hospital in North India. J Infect Dev Ctries 2012;6(7):551-4.

19. Pone SM, Hökerberg YH, de Oliveira RV, Daumas RP, Pone TM, Pone MV, et al. Clinical and laboratory signs associated to severe dengue disease in hospitalized children. J Pediatr (Rio J) 2016;92:464-71.

20. Kuo CH, Tai DI, Chang-Chien CS, Lan CK, Chiou SS, Liaw YF. Liver biochemical tests and dengue fever. Am J Trop Med Hyg 1992;47:265-70.

21. Ageep AK. Degree of liver injury in dengue virus infection. J Gen Mol Virol 2012;4(1):1-5

22. Wong M, Shen E. The utility of liver function tests in dengue. Ann Acad Med Singapore 2008;37:82-3.

23. White NJ. The treatment of malaria. N Engl J Med 1996;335:800-6.

24. Khan NA, Azhar EI, El-Fiky S, Madani HH, Abuljadial MA, Ashshi AM, et al. Clinical profile and outcome of hospitalized patients during first outbreak of dengue in Makkah, Saudi Arabia. Acta Trop 2008;105:39-44.

25. Shah I. Dengue and liver disease. Scand J Infect Dis 2008;40(11-12):993-4.

26. Hathirat $P$, Isarangkura P, Srichaikul T, Suvatte V, Mitrakul C. Abnormal hemostasis in dengue hemorrhagic fever. Southeast Asian J Trop Med Public Health 1993;24 Suppl 1:80-5.

27. Isarangkura $\mathrm{PB}$, Pongpanich $\mathrm{B}$, Pintadit $\mathrm{P}$, Phanichyakarn $\mathrm{P}$, Valyasevi A. Hemostatic derangement in dengue haemorrhagic fever. Southeast Asian J Trop Med Public Health 1987;18:331-9.

28. Horvath R, McBride WJ, Hanna J. Clinical features of hospitalized patients during Dengue-3 epidemic in far north Queensland 1997-1999. Dengue Bull 1999;23:24-9.

29. Nair VR, Unnikrishnan D, Satish B, Sahadulla MI. Acute renal failure in dengue fever in the absence of bleeding manifestations or shock. Infect Dis Clin Pract 2005;13(3):142-3.

30. Puccioni-Sohler M, Soares CN, Papaiz-Alvarenga R, Castro MJ, Peralta JM. Neurologic dengue manifestations associated with intrathecal specific immune response. Neurology 2009;27:1413-7.

31. Cam BV, Fonsmark L, Hue NB. Phuong NT, Poulsen A, Heegaard ED. Prospective case-control study of encephalopathy in children with dengue hemorrhagic fever. Am J Trop Med Hyg 2001;65(6):848-51.

32. Solomon T, Dung NM, Vaughn DW, Kneen R, Thao LT, Raengsakulrach $\mathrm{B}$, et al. Neurological manifestations of dengue infection. Lancet 2000;355:1053-9. 